\title{
Position-dependent splicing activation and repression by SR and hnRNP proteins rely on common mechanisms
}

\author{
STEFFEN ERKELENZ, ${ }^{1,3}$ WILLIAM F. MUELLER, ${ }^{2,3}$ MELANIE S. EVANS, ${ }^{2}$ ANKE BUSCH, ${ }^{2}$ KATRIN SCHÖNEWEIS, ${ }^{1}$ \\ KLEMENS J. HERTEL, ${ }^{2,4}$ and HEINER SCHAAL ${ }^{1,4}$ \\ ${ }^{1}$ Institute of Virology, Heinrich-Heine-University, D-40225 Düsseldorf, Germany \\ ${ }^{2}$ Department of Microbiology and Molecular Genetics, University of California, Irvine, Irvine, California 92697-4025, USA
}

\begin{abstract}
Alternative splicing is regulated by splicing factors that modulate splice site selection. In some cases, however, splicing factors show antagonistic activities by either activating or repressing splicing. Here, we show that these opposing outcomes are based on their binding location relative to regulated 5 ' splice sites. SR proteins enhance splicing only when they are recruited to the exon. However, they interfere with splicing by simply relocating them to the opposite intronic side of the splice site. hnRNP splicing factors display analogous opposing activities, but in a reversed position dependence. Activation by SR or hnRNP proteins increases splice site recognition at the earliest steps of exon definition, whereas splicing repression promotes the assembly of nonproductive complexes that arrest spliceosome assembly prior to splice site pairing. Thus, SR and hnRNP splicing factors exploit similar mechanisms to positively or negatively influence splice site selection.
\end{abstract}

Keywords: SR protein; hnRNP protein; pre-mRNA splicing; splicing activation; splicing repression

\section{INTRODUCTION}

Alternative pre-mRNA splicing promotes high proteomic diversity despite the limited number of protein coding genes (Nilsen and Graveley 2010). Intron removal is carried out by the spliceosome and a broad inventory of accessory proteins (Wahl et al. 2009) that recognize the boundaries of exons through sequence-specific interactions. Base-pairing between the $5^{\prime}$ end of the U1 snRNA and the exon/intron junction initiates the assembly of the spliceosome on the premRNA. The efficiency of this process depends on the complementarity between the $5^{\prime}$ splice site $\left(5^{\prime}\right.$ ss) and the $5^{\prime}$ end of the U1 snRNA (Kammler et al. 2001) as well as the presence of nearby splicing regulatory elements (SREs) (Blencowe 2006; Nilsen and Graveley 2010).

In general, SREs serve as binding sites for splicing regulatory proteins that either increase or decrease spliceosomal component recruitment (Lin and Fu 2007; Venables 2007). For example, SR proteins associated with exonic SREs to facilitate U1 snRNP recruitment to the 5 'ss through

\footnotetext{
${ }^{3}$ These authors contributed equally to this work.

${ }^{4}$ Corresponding authors

E-mail khertel@uci.edu

E-mail schaal@uni-duesseldorf.de

Article published online ahead of print. Article and publication date are at http://www.rnajournal.org/cgi/doi/10.1261/rna.037044.112.
}

their RS domain (Fu and Maniatis 1992; Lin and Fu 2007) or through RNA binding domain interactions with $\mathrm{U} 1$ snRNP-specific protein 70K (Cho et al. 2011). However, in some cases SR proteins were shown to interfere with exon selection when bound to intronic positions, potentially due to steric hindrance (Kanopka et al. 1996; Ibrahim et al. 2005; Hicks et al. 2010). hnRNP-mediated repression from exonic positions has been documented (Chen et al. 1999; Jacquenet et al. 2001; Rothrock et al. 2005), and in some cases, it was shown that an intronic hnRNP could activate splicing (Schaub et al. 2007; Wang et al. 2011). Even more, from an evolutionary viewpoint, it appears that intronic hnRNP binding sites have played a major role in shaping the landscape of mammalian SREs (Voelker et al. 2012). In combination with recently defined RNA splicing maps that correlate profiles of RNA-protein interactions with their effects on splicing activation (Ule et al. 2006; Llorian et al. 2010; Huelga et al. 2012), these observations suggest that the activity of splicing regulatory proteins might be highly position dependent.

Here we show that almost all splicing regulatory proteins tested can activate $5^{\prime}$ ss selection from defined positions. Surprisingly, all splicing regulatory proteins tested also interfere with spliceosomal recognition by simply relocating their binding sites to the opposite side of the regulated 5 'ss. The position-dependent silencing activity of splicing 
regulatory proteins does not interfere with initial U1 snRNP recruitment, but it induces the formation of nonfunctional complexes incapable of maturing into functional spliceosomes.

\section{RESULTS}

\section{The location of a SRE determines if a neighboring 5 'ss is used}

To address how the position of a SRE relative to a 5 'ss affects its recognition, a panel of known high-affinity binding sites for splicing regulatory proteins was inserted upstream of or downstream from the $5^{\prime}$ ss of a splicing reporter (Fig. 1A;

A

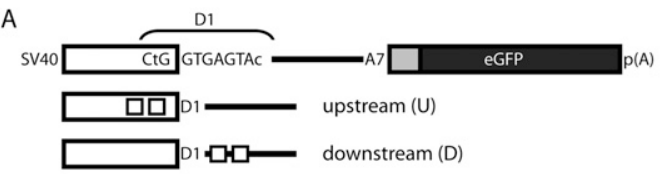

$B$ SR proteins hnRNP-like hnRNPs N1 SF2 SF7 TIA IAS Fox F/H A1 PTB $\frac{U D}{U D} \overline{U D} \overline{U D} \overline{U D} \overline{U D} \overline{U D} \overline{U D} \overline{U D}$ $\sim \sim \sim$

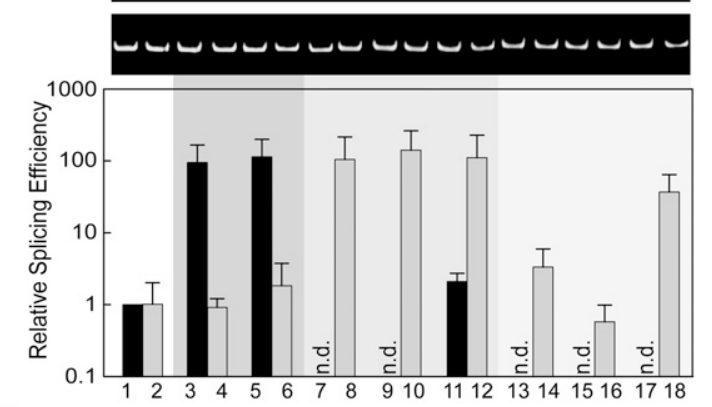

C

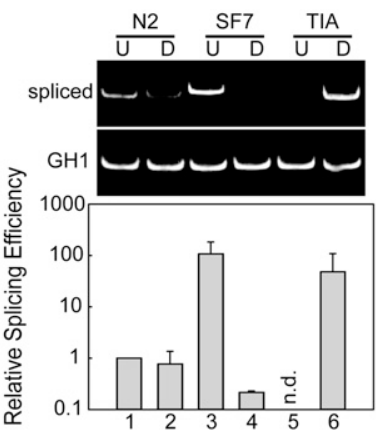

FIGURE 1. The positioning of splicing regulatory elements determines their effect on $5^{\prime}$ splice site ( $5^{\prime}$ ss) activation. (A) Schematic drawing of the RNA splicing reporter. Specific binding sites for splicing regulatory proteins are immediately upstream of or downstream from the D1 5'ss, as indicated by boxes. HeLa cells were transiently transfected with each of the reporter constructs and analyzed by semi-quantitative and real-time PCR to determine the position-dependent activity of SREs $(B)$ and to demonstrate that each SRE displays position-dependent activation and repression of splicing $(C)$. N1 and N2 are splicing neutral and near neutral control sequences (Supplemental Fig. 2). $\mathrm{U}$ and $\mathrm{D}$ refer to upstream and downstream positions relative to the D1 5'ss. n.d. refers to not detected above background. All data were normalized to the constitutively spliced GH1 mRNA.
Kammler et al. 2001; Caputi et al. 2004). By use of RT-PCR, we observed that SR proteins only activate splicing when recruited upstream of the $5^{\prime}$ ss, while hnRNP proteins activated splicing only when recruited downstream from the $5^{\prime}$ ss (Fig. 1B). These results were also validated using splicing-sensitive cell imaging (Supplemental Fig. 1). Relocating the SRSF7 SRE to the downstream position or relocating the TIA-1 SRE to the upstream position did not support 5'ss selection (Fig. 1C, lanes 3-6). In fact, 5'ss usage was repressed when the same constructs were compared with splicing neutral controls (Fig. 1C, lanes 1,2). These results show that mislocalization of SREs to the opposite side of a regulated $5^{\prime}$ ss interferes with splice site activation. We conclude that all SREs tested contribute to 5 'ss selection in a position-dependent manner, either promoting or repressing splice site recognition.

To evaluate the effects of multiple SREs, we placed SRSF7 and TIA-1 binding sites upstream of and downstream from the 5 ss, or opposing each other on either side of the $5^{\prime}$ ss. Multiple SREs located at activating positions promoted splicing of very weak 5 'ss's, supporting the notion that the overall ability of the spliceosome to recognize a $5^{\prime}$ ss depends on U1 snRNA complementarity and the strength of SREs in its neighborhood (Supplemental Fig. 3). When identical SREs were present at upstream and downstream locations, the exonic activity generally prevailed. Furthermore, if activating and repressive SREs are localized to exonic positions, the SRE closest to the 5 'ss dominated splicing decisions (Supplemental Fig. 4).

\section{MS2 tethering of splicing regulatory proteins mimics positional requirements for 5 'ss activation}

By using naturally occurring SREs, we formally could not exclude the possibility that the SRE itself modulated 5'ss activation or that other factors interact with the SRE. We therefore evaluated position-dependent splicing using an MS2 tethering system. Placing SR protein fusion proteins immediately upstream of the 5 'ss promoted splicing, whereas SR fusion recruitment to the downstream intronic position resulted in splicing repression (Fig. 2A). In contrast, hnRNP fusion proteins stimulated splicing only when they were tethered to the downstream intron. When localized to the opposite side of the $5^{\prime}$ ss, hnRNP fusion proteins actively repressed intron excision. In summary, the tethering experiments confirm the conclusion that the activities of splicing regulatory proteins are highly position dependent.

To determine the effects of splicing regulatory proteins on the selection of two alternative $5^{\prime}$ ss's, we inserted tandem MS2 RNA hairpins between two identical 5 'ss sequences (Fig. 2B). All SR fusion proteins promoted splicing of the downstream $5^{\prime}$ ss, whereas all hnRNP factors tested favored the upstream 5'ss (Fig. 2B). Consistent with these results, the natural binding site for SRSF7 showed a strong preference 


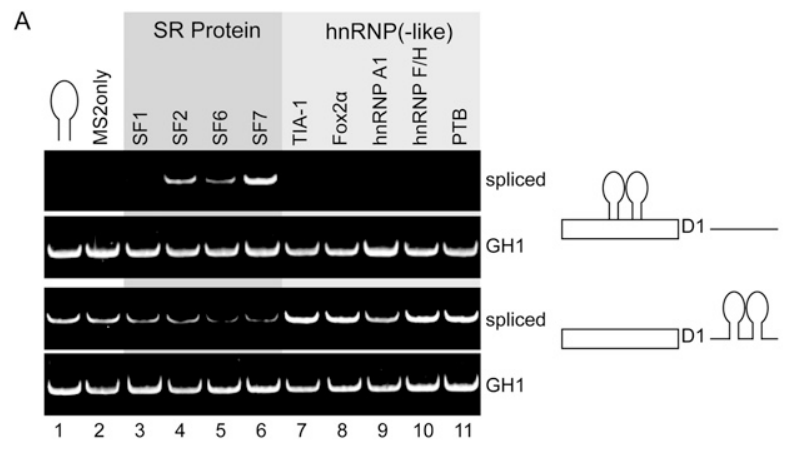

B

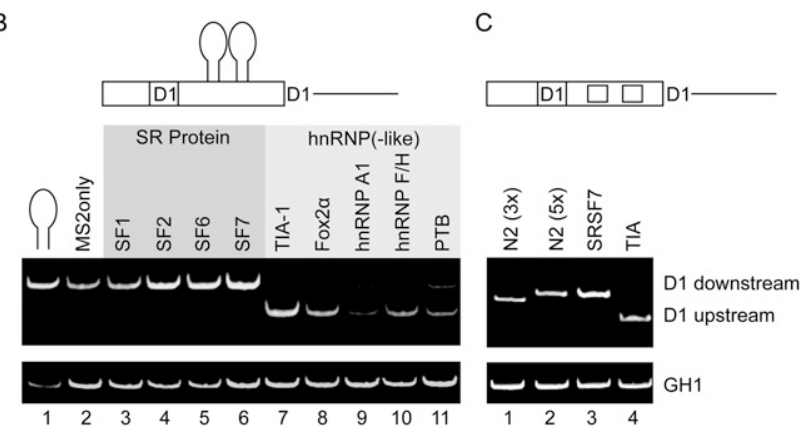

FIGURE 2. MS2 tethering of splicing regulatory proteins mimics positional requirements for 5 ss activation. (A) Expression of MS2 fusion proteins results in position-dependent activation or repression of splicing using reporters that harbor MS2 binding sites upstream of or downstream from the D1 5'ss. Comparable expression of all MS2 fusion proteins was verified by Western blot analysis (Supplemental Fig. 5). (B) Schematic diagram of the splicing reporter in which the two MS2 binding sites are flanked by the identical up- and downstream 5 'ss D1. The splicing pattern of each sample was analyzed using semiquantitative RT-PCR analysis. $(C)$ Identical experiments were performed using splicing reporters where the MS2 binding sites were replaced by high-affinity binding sites for endogenous TIA-1 and SRSF7.

for activating the downstream $5^{\prime}$ ss, whereas binding sites for TIA-1 activate the upstream 5'ss (Fig. 2C).

\section{The progression into a functional splicing complex depends on the side from which U1 snRNP is recruited to the $5^{\prime}$ ss}

To determine the mechanisms of splicing repression by intronic SR proteins, we measured intron removal efficiency of a $\beta$-globin-based pre-mRNA substrate that contains a single MS2 RNA hairpin downstream from its $5^{\prime}$ ss. In agreement with the cell culture experiments, splicing of this substrate was impaired when SRSF7 was tethered downstream from the 5 'ss (Fig. 3A,B). When placed upstream of the regulated 5'ss, SRSF7 activated splicing. The parallel but opposite effect was observed for TIA-1 (Fig. $3 \mathrm{~A}, \mathrm{~B})$, which repressed from an exonic location but activated from an intronic location. It is worth noting here that the splicing reporters used in the cell transfection and in the in vitro splicing experiments are unrelated, thus supporting the notion that the position-dependent splicing activities are not reporter specific but are perhaps more general in nature.

To determine the mechanisms of the observed splicing repression, we uncoupled the ATP-dependent and ATPindependent steps of splicing through the use of an E-complex pulse chase experiment (Kotlajich et al. 2009). Splicing reactions were enriched for E-complex in the absence of ATP and then chased to spliced products. When MS2SRSF7 was located within the intron, only $10 \%$ of the E-complexes converted into spliced mRNAs. However, the rate of splicing for this small fraction was essentially identical to the rates measured in the absence of MS2SRSF7 (Supplemental Fig. 6). These results show that only a small portion of splicing-competent E-complexes was formed on the pre-mRNA in the presence of intronic MS2-SRSF7 and that this small fraction behaves kinetically similar to uninhibited pre-mRNAs.

To determine if spliceosomal complex formation was altered when splicing was repressed, we analyzed complex formation in the presence and absence of MS2-SRSF7 and MS2-TIA-1. Neither protein, when localized to their repressive position, interfered with spliceosome assembly at the early E-complex stage, indicating that U1 snRNP is efficiently recruited to the pre-mRNA substrate (Fig. 3C). However, this initial U1 snRNP recruitment appears to be a "dead-end" E-complex because further incubation in the presence of ATP led to less formation of higher-order complexes (A-, B-, and C-complex) (Fig. 3D). These results demonstrate that the presence of SRSF7 or TIA-1 at the repressive positions accumulate splicing-incompetent E-complexes that do not efficiently progress into functional spliceosomes. We conclude that U1 snRNP is recruited efficiently to the pre-mRNA regardless of the presence of an inhibitory SRE. However, the presence of the inhibitory SRE interferes with the progression into later stages of spliceosome assembly.

Based on these observations, it is expected that U1 snRNP components interact with the pre-mRNA substrate, independent of SRE location. To confirm this prediction, we tested for the presence of $\mathrm{U} 1-70 \mathrm{~K}$ and $\mathrm{U} 1-\mathrm{C}$ upon immobilizing in vitro transcribed RNAs containing a 5'ss and SREs at various locations. While the splicing near neutral control precipitated only low levels of U1 snRNPspecific proteins (Fig. 4A, lane 3), we detected high levels of U1-70K and U1-C on RNAs containing the SRSF7 binding site upstream of or downstream from the 5 'ss (Fig. 4A, lanes 4,5). These results confirm the prediction that the splicing repressive phenotype of SRSF7 is not caused by an interference to recruit U1 snRNP to the 5 'ss. A slightly different U1 snRNP recruitment trend was observed for RNAs containing TIA-1 binding sites. Increased levels of U1-70K and U1-C were detected on RNAs containing the TIA-1 binding site in its activating intronic position (Fig. $4 \mathrm{~A}$, cf. lanes 3 and 7). However, when placed into a splicing repressive position, TIA-1 binding did not increase levels of 
A

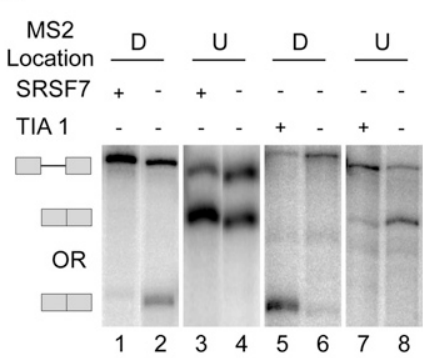

B

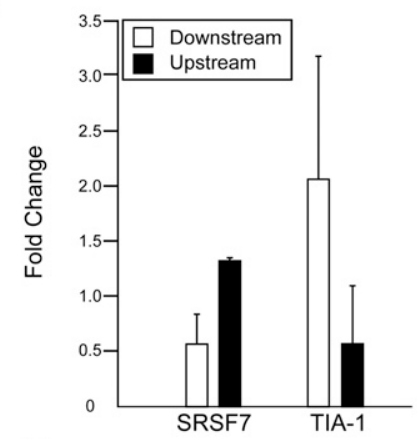

C
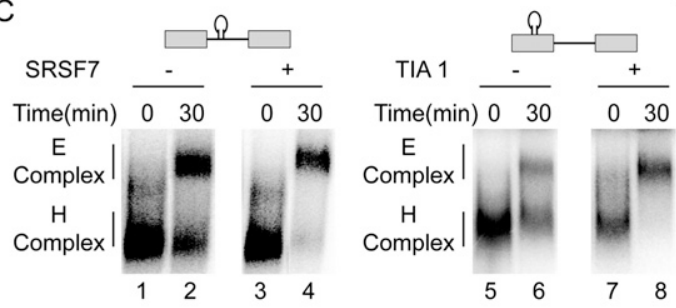

D
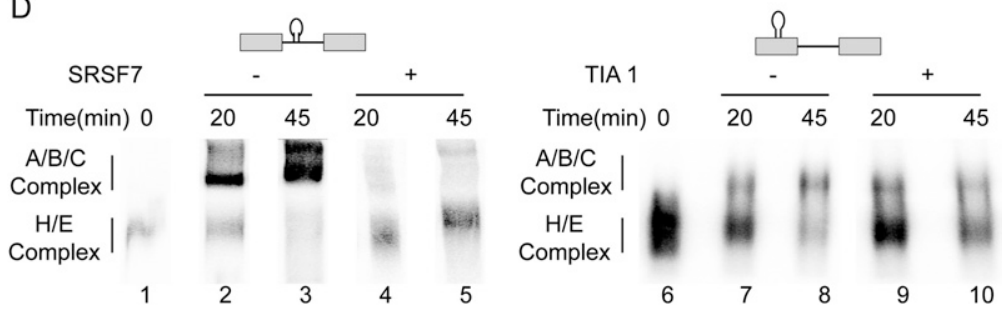

FIGURE 3. Binding of SRSF7 to the intron does not interfere with E-complex formation. $(A)$ In vitro splicing reaction time course in the presence or absence of MS2-RS-SRSF7 or MS2TIA-1 binding either upstream of or downstream from the 5 'ss. $(B)$ In vitro splicing reactions were repeated and quantified. $(C)$ ATP-independent E-complex formation in the presence or absence of MS2-RS-SRSF7 or MS2-TIA-1 was visualized using agarose gel electrophoresis. $(D)$ Higher-order, ATP-dependent complex formation in the presence or absence of MS2-RSSRSF7 or MS2-TIA-1 was visualized using polyacrylamide native gels or agarose gels. Spliceosomal complexes or splicing products formed during the reactions are defined next to each gel.

U1 snRNP recruitment. Rather, the presence of TIA-1 in the repressive exonic position does not interfere with the intrinsic binding of U1 snRNP to the D1 5'ss (Fig. 4A, cf. lanes 3 and 6). Together, these results suggest that SR and hnRNP proteins act through similar mechanisms to activate splicing by increasing U1 snRNP recruitment. When located in repressive positions, SR and hnRNP proteins do not interfered with U1 snRNP recruitment. However, higher-order spliceosomal complex formation is impaired at the stage of A-complex formation.

To determine whether "dead-end" E-complex formation depends on U1 snRNA interactions with the pre-mRNA, we incapacitated U1 snRNA binding by oligonucleotidedirected RNase $\mathrm{H}$ digestion of the $\mathrm{U} 1$ snRNA $5^{\prime}$ end. In all SRE permutations tested, U1 snRNP recruitment was severely reduced by the loss of the $5^{\prime}$ end of U1 snRNA, demonstrating that U1 snRNP recruitment requires RNA duplex formation (Fig. 4B). Furthermore, identical immobilization experiment using RNAs that harbor a mutated 5'ss showed that a functional $5^{\prime}$ ss is required for efficient recruitment of spliceosomal components (Fig. 4C). Together, these results demonstrate that base-pairing between the U1 snRNA and the 5 'ss is required for $\mathrm{U} 1 \mathrm{snRNP}$ recruitment to the pre-mRNA but that the ability of the E-complex to progress into spliceosomal A-, B-, and C-complexes is regulated by surrounding SREs. Our findings show that positioning SR proteins to the downstream intron, or hnRNPs to the upstream exon, represses splicing without significantly altering initial recruitment of $U 1$ snRNP to the pre-mRNA.

Based on these results, it is expected that constitutive exons should display a higher frequency of SREs in activating positions compared with alternatively spliced exons. To test this hypothesis, we determined the frequency of SREs in exonic or intronic positions of human exons within a 30 -nucleotide (nt) window upstream of and downstream from their 5'ss. Indeed, constitutive exons are more likely to harbor an upstream SRSF7 or a downstream TIA-1 binding site compared with alternative exons (Supplemental Table 1). In contrast, alternative exons display a higher frequency of intronic SRSF7 and exonic TIA-1 binding sites. This analysis can be extended to other hnRNPs, demonstrating that constitutive exons generally display an increased frequency of hnRNP binding sites in the activating intronic position (Supplemental Table 2). Furthermore, knockdown of individual hnRNPs, as recently reported (Huelga et al. 2012), recorded changes in splicing efficiencies of exons that were identified by our motif search as potential hnRNP targets (data not shown).

\section{DISCUSSION}

Here we show that the rigid categorization of SREs into enhancers or repressors should be re-evaluated. Based on our results, we propose a model in which the positioning of a SRE relative to a $5^{\prime}$ ss determines whether the formation of splicing complexes is productive or not. While a repressing SRE does not interfere with the recruitment of U1 snRNP to the regulated 5 'ss, it traps early spliceosomal complexes in an inactive state, potentially resulting in the recruitment of qualitatively distinct U1 snRNPs that may differ in their protein composition (Hernandez et al. 2009) or U1 snRNA (Kyriakopoulou et al. 2006). 
A

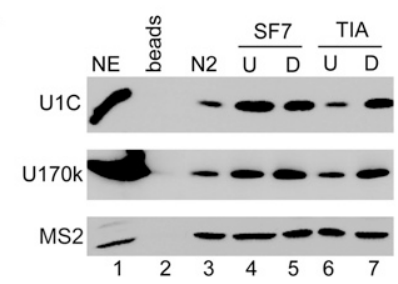

B

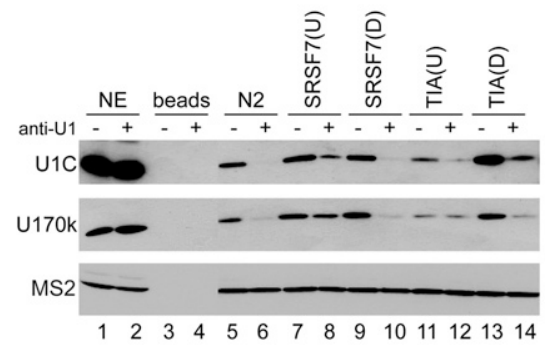

C

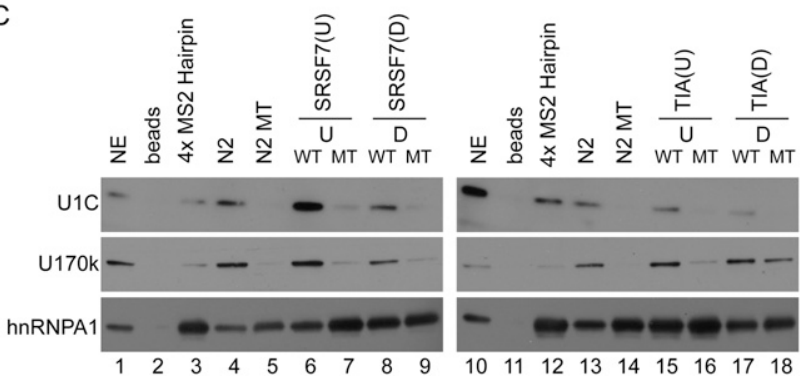

FIGURE 4. The recruitment of U1 snRNP components to the 5 'ss is not decreased by repressive SREs. (A) RNAs were immobilized using Agarose beads and analyzed for the presence of U1 snRNP with specific antibodies directed against U1-70K and U1-C. MS2 coat protein was used as a loading control. N2 represents the splicing near neutral control sequence. (B) HeLa cell nuclear extracts were depleted (D) or mock depleted (U) of functional U1 snRNP using short DNA oligonucleotides and RNase $\mathrm{H}$. The extracts were then used in RNA pulldown assays to demonstrate that U1 snRNA/RNA interactions are necessary for U1 snRNP recruitment. MS2 coat protein and hnRNP A1 were used to control for loading, and N2 represents splicing near neutral control sequences. (C) RNAs containing a muted 5'ss were used in the pulldown assay to demonstrate the effect of a functional or nonfunctional splice site on U1 recruitment.

\section{SR and hnRNP proteins have activities that differ depending on their position relative to the regulated splice site}

For SRE-dependent splice site recognition, a mechanistic picture emerges that indicates surprising similarities between the actions of SR and hnRNP proteins. Both classes of splicing factors demonstrate position-dependent activation or repression. As has been demonstrated previously, SR proteins bound to exons recruit the splicing machinery to splice sites (Graveley 2000; Black 2003; Cho et al. 2011). The observed SR protein splicing repression is based on stalling the progression of spliceosomal assembly after efficient formation of the E-complex. Thus, the recruited components are not able to efficiently establish splice site pairing, a process mediated by RS-domain containing bridging factors Prp5 and Rsd1 (Shao et al. 2012). It is possible that intronic SR proteins may prevent the interaction between pre-mRNA-bound U1 and U2 snRNPs by competing for Prp5 (at the 3' splice site) or Rsd1 (at the $5^{\prime}$ ss) binding. Alternatively, the correct placement of U1 snRNP at the 5'ss could be a measure for rearranging the conformational state of the particle to promote the recruitment of other splicing factors required for further spliceosomal assembly, similar in principle with the demonstration that alternate conformations of U2AF65 promote spliceosome assembly (Mackereth et al. 2011).

The effects of hnRNP proteins on 5 'ss activation are mirror images of SR protein activities. When bound to exons, they repress splicing, and when bound to introns, they activate splicing. Interestingly, all hnRNP proteins tested follow this antagonistic activity profile (with the exception of hnRNP A1: No evidence has yet been presented that hnRNP A/B proteins recruit spliceosomal components; this may mean they interact with the spliceosome differently than the other hnRNPs) (Dreyfuss et al. 1993; Mayeda et al. 1998). Activating hnRNP positions promote E-complex assembly; however, splicing repression from an exonic position did not interfere with initial 5 'ss recognition. Thus, splicing suppression is achieved after initial splice site recognition regardless of whether the repression is exerted by exonic hnRNPs or intronic SR proteins. These observations are consistent with studies demonstrating that splicing silencer motifs impair the commitment to splice site pairing (Yu et al. 2008). Splice site activation by hnRNPs follows the mechanisms worked out for classical exonic enhancers, increased recruitment of U1 snRNP to the 5 'ss. In summary, SR and hnRNP splicing regulatory proteins appear to target the same steps to promote or interfere with spliceosomal assembly.

While the splicing promoting and repressing activities of SR proteins can be explained by RS-domain interactions, it is unclear how hnRNP splicing regulators increase U1 snRNP recruitment or interfere with prespliceosomal complex formation. Activation by intronic TIA-1 has been demonstrated to target the U1-C component of U1 snRNP (Forch et al. 2002), suggesting that other intronically bound hnRNPs may use similar interactions. Exonic hnRNPs may stabilize cross-exon interactions, thus reducing the efficiency of splice site pairing (Motta-Mena et al. 2010).

The rescue of very weak 5 ss when embedded within a context of activating SRE positions highlights the shortcomings of present in silico approaches that usually ignore the sequence environment of $5^{\prime}$ ss for prediction of their functionality. Our studies illustrate that the position of SREs relative to a 5 'ss defines their splicing phenotype, providing a mechanistic framework for exonic and intronic splicing repression/activation. Integrating these new insights into current splicing code algorithms will likely improve the success rate of splice pattern predictions. 


\section{MATERIALS AND METHODS}

\section{Plasmids}

All transfection constructs are based on the HIV-1 glycoprotein/ eGFP expression plasmid (Kammler et al. 2001). All upstream and downstream splicing elements (Supplemental Table 3) were cloned into the reporter using PCR-product using a specific forward and reverse primer pairs (please contact corresponding authors for primer sequences). MS2 fusion protein expressing plasmids were generated according to the method described previously (Singh et al. 2010).

\section{Cell culture and RT-PCR analysis}

HeLa cells were maintained in DMEM supplemented with $10 \%$ fetal calf serum. Total RNA was collected $30 \mathrm{~h}$ after transfection, reverse transcribed, and analyzed by qPCR or semi-quantitative PCR. All experiments were repeated three times.

\section{In vitro splicing and complex formation}

Reactions were carried out in 30\% nuclear extract containing ${ }^{32} \mathrm{P}$-labeled RNA transcripts with or without saturating amounts of recombinant MS2-RS-SRSF7 (250 nM) or MS2-TIA-1 (500 nM), according to the method previously described (Hicks et al. 2005). Pulse chase experiments were carried out according to the method previously described (Konarska and Sharp 1986; Kotlajich et al. 2009). ATP-independent complex formation reactions were carried out like the splicing reactions except no ATP or creatine phosphate was added to the reaction. Following incubation, reactions were loaded onto a 1.5\% low-melt Agarose gel and analyzed by PhosphorImager analysis (Das and Reed 1999; Hicks et al. 2005). ATP-dependent complex formation was analyzed according to the method previously described (Konarska and Sharp 1986; Kotlajich et al. 2009).

\section{Immobilization of RNA on Agarose beads and RNA affinity assays}

Substrate RNAs were covalently linked to adipic acid dihydrazideAgarose beads according to the method previously described (Kammler et al. 2006). Immobilized RNAs were incubated in 30\% HeLa cell nuclear extract/buffer D for $30 \mathrm{~min}$ at $30^{\circ} \mathrm{C}$. Beads were then washed, and proteins were eluted by the addition of $2 \times$ protein sample buffer. Proteins were resolved by SDS-PAGE and were subjected to immunoblot analysis (see Protein Analysis section).

\section{Protein analysis}

Transfected cells were lysed in RIPA buffer. Proteins were separated by SDS-PAGE and subjected to immunoblotting. Membranes were probed with rabbit antibody against HA (H6908), mouse antibody against $\beta$-actin (A2228), rat antibody against U1-C (4H12) (all Sigma-Aldrich), goat antibody against U1-70K (Santa Cruz Biotechnology, C-18), mouse antibody against hnRNP A1 (Santa Cruz Biotechnology, 9H10), and rabbit antibody against MS2 coat protein (Tetracore, TC-7004).

\section{Computational analysis}

To determine the frequency of potential SRE binding sites upstream of and/or downstream from known splice sites within the human genome, a list of constitutive (99885) and alternative 5 'ss (5554) exons was compiled based on known mRNA isoforms and EST information (GRCh37/hg19) (Kent et al. 2002). To calculate frequencies of potential TIA-1 and SRSF7 binding sites, the surrounding 30-nt sequence space was scanned for occurrences of either TTTTT or at least two occurrences of TTT in the case of TIA-1 (Dember et al. 1996) and for occurrences of [A/T]C[A/T][A/T]C in the case of SRSF7 (Cavaloc et al. 1999). $P$-values were calculated using Fisher's exact test, comparing the frequency of each motif combination (in both groups) with the sum of the frequencies of any other combination. To determine the effect of hnRNP knockdowns on directional splicing events, we used a list of exons that change their splicing behavior upon knockdown of hnRNP A1 and hnRNP F, respectively, which were kindly provided by Gene Yeo (Huelga et al. 2012). For all exons in our list, we extracted the sequence of $30 \mathrm{nt}$ upstream of and downstream from the 5 'ss. Those sequences were scanned for occurrences of potential binding sites for hnRNP Al (TTAGGG) and hnRNP F (AGGGA), respectively.

\section{SUPPLEMENTAL MATERIAL}

Supplemental material is available for this article.

\section{ACKNOWLEDGMENTS}

We thank John Conboy, Juan Valcárcel, Massimo Caputi, Brenton Graveley, Chris Smith, Alain Cochrane, and David Peabody for providing plasmids. We thank Björn Wefers, Eva Leimbach, Christin Bruchhagen, Christiane Breuer, Tomasz Ochman, and Sarah Rosin for excellent technical assistance. This work was supported by grants from the Stiftung für AIDS-Forschung, Düsseldorf (H.S.), the Jürgen-Manchot-Stiftung (H.S.), Boehringer Ingelheim Fonds (travel allowance to S.E.), NIH (RO1 GM62287 and R21 CA149548 to K.J.H.), and a fellowship within the Postdoc Programme of the German Academic Exchange Service, DAAD (A.B.).

Received October 26, 2012; accepted October 30, 2012.

\section{REFERENCES}

Black DL. 2003. Mechanisms of alternative pre-messenger RNA splicing. Annu Rev Biochem 72: 291-336.

Blencowe BJ. 2006. Alternative splicing: new insights from global analyses. Cell 126: 37-47.

Caputi M, Freund M, Kammler S, Asang C, Schaal H. 2004. A bidirectional SF2/ASF- and SRp40-dependent splicing enhancer regulates human immunodeficiency virus type $1 \mathrm{rev}$, env, $v p u$, and nef gene expression. J Virol 78: 6517-6526.

Cavaloc Y, Bourgeois CF, Kister L, Stevenin J. 1999. The splicing factors 9G8 and SRp20 transactivate splicing through different and specific enhancers. RNA 5: 468-483.

Chen CD, Kobayashi R, Helfman DM. 1999. Binding of hnRNP H to an exonic splicing silencer is involved in the regulation of alternative splicing of the rat $\beta$-tropomyosin gene. Genes Dev 13: 593-606.

Cho S, Hoang A, Sinha R, Zhong XY, Fu XD, Krainer AR, Ghosh G. 2011. Interaction between the RNA binding domains of Ser-Arg splicing factor 1 and U1-70K snRNP protein determines early spliceosome assembly. Proc Natl Acad Sci 108: 8233-8238. 
Das R, Reed R. 1999. Resolution of the mammalian E complex and the ATP-dependent spliceosomal complexes on native agarose minigels. RNA 5: 1504-1508.

Dember LM, Kim ND, Liu KQ, Anderson P. 1996. Individual RNA recognition motifs of TIA-1 and TIAR have different RNA binding specificities. J Biol Chem 271: 2783-2788.

Dreyfuss G, Matunis MJ, Pinol-Roma S, Burd CG. 1993. hnRNP proteins and the biogenesis of mRNA. Annu Rev Biochem 62: 289321.

Forch P, Puig O, Martinez C, Seraphin B, Valcarcel J. 2002. The splicing regulator TIA-1 interacts with U1-C to promote U1 snRNP recruitment to $5^{\prime}$ splice sites. EMBO J 21: 6882-6892.

Fu XD, Maniatis T. 1992. The $35-\mathrm{kDa}$ mammalian splicing factor SC35 mediates specific interactions between U1 and U2 small nuclear ribonucleoprotein particles at the $3^{\prime}$ splice site. Proc Natl Acad Sci 89: 1725-1729.

Graveley BR. 2000. Sorting out the complexity of SR protein functions. RNA 6: 1197-1211.

Hernandez H, Makarova OV, Makarov EM, Morgner N, Muto Y, Krummel DP, Robinson CV. 2009. Isoforms of U1-70k control subunit dynamics in the human spliceosomal U1 snRNP. PLoS ONE 4: e7202. doi: 10.1371/journal.pone.0007202.

Hicks MJ, Lam BJ, Hertel KJ. 2005. Analyzing mechanisms of alternative pre-mRNA splicing using in vitro splicing assays. Methods 37: 306-313.

Hicks MJ, Mueller WF, Shepard PJ, Hertel KJ. 2010. Competing upstream $5^{\prime}$ splice sites enhance the rate of proximal splicing. Mol Cell Biol 30: 1878-1886.

Huelga SC, Vu AQ, Arnold JD, Liang TY, Liu PP, Yan BY, Donohue JP, Shiue L, Hoon S, Brenner S et al. 2012. Integrative genomewide analysis reveals cooperative regulation of alternative splicing by hnRNP proteins. Cell Rep 1: 167-178.

Ibrahim EC, Schaal TD, Hertel KJ, Reed R, Maniatis T. 2005. Serine/arginine-rich protein-dependent suppression of exon skipping by exonic splicing enhancers. Proc Natl Acad Sci 102: 5002-5007.

Jacquenet S, Mereau A, Bilodeau PS, Damier L, Stoltzfus CM, Branlant C. 2001. A second exon splicing silencer within human immunodeficiency virus type 1 Tat exon 2 represses splicing of Tat mRNA and binds protein hnRNP H. J Biol Chem 276: 40464 40475.

Kammler S, Leurs C, Freund M, Krummheuer J, Seidel K, Tange TO, Lund MK, Kjems J, Scheid A, Schaal H. 2001. The sequence complementarity between HIV-1 5' splice site SD4 and U1 snRNA determines the steady-state level of an unstable env pre-mRNA. RNA 7: 421-434.

Kammler S, Otte M, Hauber I, Kjems J, Hauber J, Schaal H. 2006. The strength of the HIV-1 $3^{\prime}$ splice sites affects Rev function. Retrovirology 3: 89. doi: 10.1186/1742-4690-3-89.

Kanopka A, Muhlemann O, Akusjarvi G. 1996. Inhibition by SR proteins of splicing of a regulated adenovirus pre-mRNA. Nature 381: 535-538.

Kent WJ, Sugnet CW, Furey TS, Roskin KM, Pringle TH, Zahler AM, Haussler D. 2002. The human genome browser at UCSC. Genome Res 12: 996-1006.

Konarska MM, Sharp PA. 1986. Electrophoretic separation of complexes involved in the splicing of precursors to mRNAs. Cell 46: $845-855$.
Kotlajich MV, Crabb TL, Hertel KJ. 2009. Spliceosome assembly pathways for different types of alternative splicing converge during commitment to splice site pairing in the A complex. Mol Cell Biol 29: $1072-1082$.

Kyriakopoulou C, Larsson P, Liu L, Schuster J, Soderbom F, Kirsebom LA, Virtanen A. 2006. U1-like snRNAs lacking complementarity to canonical 5' splice sites. RNA 12: 1603-1611.

Lin S, Fu XD. 2007. SR proteins and related factors in alternative splicing. Adv Exp Med Biol 623: 107-122.

Llorian M, Schwartz S, Clark TA, Hollander D, Tan LY, Spellman R, Gordon A, Schweitzer AC, de la Grange P, Ast G, et al. 2010. Position-dependent alternative splicing activity revealed by global profiling of alternative splicing events regulated by PTB. Nat Struct Mol Biol 17: 1114-1123.

Mackereth CD, Madl T, Bonnal S, Simon B, Zanier K, Gasch A, Rybin V, Valcarcel J, Sattler M. 2011. Multi-domain conformational selection underlies pre-mRNA splicing regulation by U2AF. Nature 475: 408-411.

Mayeda A, Munroe SH, Xu RM, Krainer AR. 1998. Distinct functions of the closely related tandem RNA-recognition motifs of hnRNP A1. RNA 4: 1111-1123.

Motta-Mena LB, Heyd F, Lynch KW. 2010. Context-dependent regulatory mechanism of the splicing factor hnRNP L. Mol Cell 37: 223-234.

Nilsen TW, Graveley BR. 2010. Expansion of the eukaryotic proteome by alternative splicing. Nature 463: 457-463.

Rothrock CR, House AE, Lynch KW. 2005. HnRNP L represses exon splicing via a regulated exonic splicing silencer. EMBO J 24: 27922802.

Schaub MC, Lopez SR, Caputi M. 2007. Members of the heterogeneous nuclear ribonucleoprotein $\mathrm{H}$ family activate splicing of an HIV-1 splicing substrate by promoting formation of ATP-dependent spliceosomal complexes. J Biol Chem 282: 13617-13626.

Shao W, Kim HS, Cao Y, Xu YZ, Query CC. 2012. A U1-U2 snRNP interaction network during intron definition. Mol Cell Biol 32: 470-478.

Singh KK, Erkelenz S, Rattay S, Dehof AK, Hildebrandt A, SchulzeOsthoff K, Schaal H, Schwerk C. 2010. Human SAP18 mediates assembly of a splicing regulatory multiprotein complex via its ubiquitin-like fold. RNA 16: 2442-2454.

Ule J, Stefani G, Mele A, Ruggiu M, Wang X, Taneri B, Gaasterland T, Blencowe BJ, Darnell RB. 2006. An RNA map predicting Novadependent splicing regulation. Nature 444: 580-586.

Venables JP. 2007. Downstream intronic splicing enhancers. FEBS Lett 581: 4127-4131.

Voelker RB, Erkelenz S, Reynoso V, Schaal H, Berglund JA. 2012. Frequent gain and loss of intronic splicing regulatory elements during the evolution of vertebrates. Genome Biol Evol 4: 659-674.

Wahl MC, Will CL, Luhrmann R. 2009. The spliceosome: design principles of a dynamic RNP machine. Cell 136: 701-718.

Wang E, Mueller WF, Hertel KJ, Cambi F. 2011. G Run-mediated recognition of proteolipid protein and DM20 5' splice sites by U1 small nuclear RNA is regulated by context and proximity to the splice site. J Biol Chem 286: 4059-4071.

Yu Y, Maroney PA, Denker JA, Zhang XH, Dybkov O, Luhrmann R, Jankowsky E, Chasin LA, Nilsen TW. 2008. Dynamic regulation of alternative splicing by silencers that modulate $5^{\prime}$ splice site competition. Cell 135: 1224-1236. 
RNA 19: 96-102 (2013)

\title{
Position-dependent splicing activation and repression by SR and hnRNP proteins rely on common mechanisms
}

\author{
STEFFEN ERKELENZ, WILLIAM F. MUELLER, MELANIE S. EVANS, ANKE BUSCH, KATRIN SCHÖNEWEIS, \\ KLEMENS J. HERTEL, and HEINER SCHAAL
}

In the above-mentioned article, Supplemental Figures 3 and 6 included several errors which have been corrected in Revised Supplemental Material online. The changes:

1. The legend presented with the original Supplemental Figure 3 was intended for a different figure and should instead read as follows:

Supplemental Figure 3: Multiple PDEs synergize in activating weak 5' splice sites. To analyze the functional interdependence of the intrinsic strength of a $5^{\prime}$ ss defined by its ability to basepair with the U1 snRNA and the sequence surrounding, we mutated the $5^{\prime}$ splice site to gradually vary its strength (A) and tested it in the context of different numbers of enhancer binding sites in the vicinity. (B) Transfection experiments were carried out as described above and samples were subjected to semi-quantitative and quantitative RT-PCR analysis.

2. The axes in panel $A$ in the original Supplemental Figure 6 were off by a factor of 10 (see Revised Supplemental, online).

The authors apologize for any confusion this may have caused. 

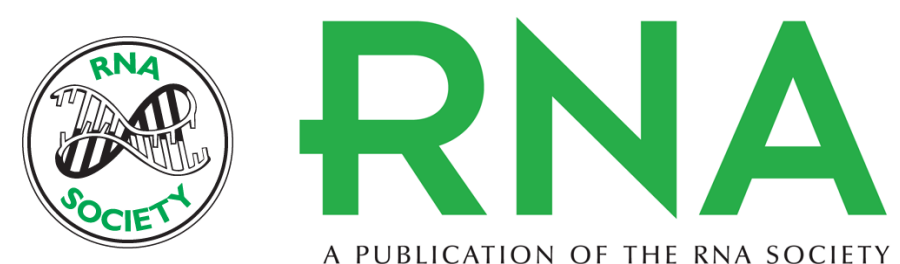

A PUBLICATION OF THE RNA SOCIETY

\section{Position-dependent splicing activation and repression by SR and hnRNP proteins rely on common mechanisms}

Steffen Erkelenz, William F. Mueller, Melanie S. Evans, et al.

RNA 2013 19: 96-102 originally published online November 21, 2012

Access the most recent version at doi:10.1261/rna.037044.112

\section{Supplemental http://rnajournal.cshlp.org/content/suppl/2012/11/08/rna.037044.112.DC1 \\ Material http://rnajournal.cshlp.org/content/suppl/2013/06/17/rna.037044.112.DC3}

Related Content Position-dependent splicing activation and repression by SR and hnRNP proteins rely on common mechanisms

Steffen Erkelenz, William F. Mueller, Melanie S. Evans, et al.

RNA July , 2013 19: 1015

References This article cites 41 articles, 21 of which can be accessed free at:

http://rnajournal.cshlp.org/content/19/1/96.full.html\#ref-list-1

Articles cited in:

http://rnajournal.cshlp.org/content/19/1/96.full.html\#related-urls

\section{License}

Email Alerting Receive free email alerts when new articles cite this article - sign up in the box at the Service top right corner of the article or click here.

\section{|||||||| Providing Precise Solutions for your research.}

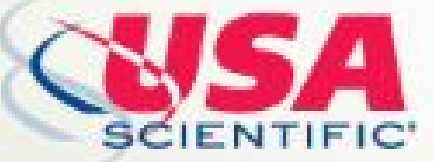

To subscribe to $R N A$ go to:

http://rnajournal.cshlp.org/subscriptions 